\title{
Impact of Supplier's R\&D Investment on Supply Chain Operations under Stochastic Isoelastic Demand and Consignment Contract
}

\author{
Hengyun Zhang ${ }^{1,2, a}$ and Ruoyu $\mathrm{Lu}^{1, \mathrm{~b}}$ \\ ${ }^{1}$ School of Management and Economics, University of Electronic Science and Technology of China, \\ Chengdu, China; \\ 2 Department of Information Engineering, Sichuan Staff University of Science and Technology, \\ Chengdu, China \\ azhyuestc@163.com, blury@uestc.edu.cn
}

Keywords: decentralized supply chain; R\&D investment; cost reduction; isoelastic stochastic demand, consignment contract.

\begin{abstract}
In a decentralized supply chain with an upstream supplier and a downstream platform, the supplier sells a product to a stochastic isoelastic demand market through a platform. The supplier considers to make R\&D investment to reduce its production cost, and hence the supply chain becomes more cost efficient. We first characterize the equilibrium pricing and production decisions for the two chain members, under any given R\&D investment strategy. We then analyze the impact of the supplier's R\&D investment on equilibrium decisions and corresponding profits of the two chain members. We find that the supplier's R\&D investment can lead to a lower retail price and a higher production quantity, and always benefit the platform's profit.
\end{abstract}

\section{Introduction}

In reality, it is quite common that firms conduct research and development (R\&D) activities to reduce their production costs, or improve their product quality. For example, in pharmaceutical industry, R\&D expenditures have increased from \$15.2 billion in 1998 to \$20.9 billion in 2001 (Grossmann, 2004). Moreover, recent studies show that in automobile industry, more than \$100 billion has been spent in R\&D activities each year (Zhang and Hong, 2016).

The existing studies on $R \& D$ activities have mainly assume that $R \& D$ investment can either lead to cost reduction or result in quality improvement. For example, assuming that R\&D activities can lead to cost reduction, Ge et al. (2014) and Usta et al. (2014) investigate R\&D investment in a one-to-one supply chain. Banerjee and Lin (2001, 2003) study R\&D investment in a one-to-many supply chain. Bernstein and Kök (2009) and Bernstein et al. (2015) explore R\&D investment in a many-to-one supply chain. Ishii (2004) and Gupta (2008) consider R\&D investment in a many-to-many supply chain. The above studies on R\&D investment assume that R\&D activities can lead to cost reduction for the firms. Assuming that R\&D activities can result in quality improvement for the firms, Leng et al. (2016) and Seifbarghy et al. (2015) study R\&D investment in a one-to-one supply chain. Chen et al. (2015) and Zhu and He (2017) consider R\&D investment in a one-to-many supply chain. Yu and Ma (2013) investigate R\&D investment in a many-to-one supply chain.

Although the existing studies have investigated R\&D investment from different perspectives, they do not explore $R \& D$ investment in a supply chain with isoelastic demand, That is, they ignore that many products may have constant price elasticities. As a matter of fact, it is estimated that the many products have constant price elasticities (for example, price elasticity for Chevrolet automobiles is 4.0, see, e.g., Anderson and McLellan 1997). In addition, they mainly assume that suppliers sell their products to retailers through wholesale price contract, and do not consider the problem that suppliers may sell products to the market through consignment contract. However, with the rapid development of platform sale, many suppliers sell their products on the platform using consignment contract.

In this paper, we consider a supply chain with a single upstream supplier and a single downstream platform. The supplier first makes R\&D investment to reduce its production cost and then sells the 
product on the platform to a isoelastic stochastic demand market. Using backward induction, we derive the optimal pricing and production decisions for the supplier and the platform. The impact of the investment on the supply chain operations is also analyzed.

In summary, the key contributions of our paper are as follows:

(1) We establish a model to address the problem of the supplier's R\&D investment in a decentralized supply chain with stochastic isoelastic demand under consignment contract.

(2) We characterize the optimal pricing and production decisions for the supplier and the optimal revenue share decision for the platform.

(3) We analyze the impact of the supplier's R\&D investment on the optimal decisions and the corresponding profits of the two chain members.

The rest of this paper is organized as follows. In Section 2, we present our model. In Section 3, we derive the optimal decisions and strategies for the two chain members. Section 4 concludes our work.

\section{The Model}

Consider a supply chain, which consists of an upstream supplier and a downstream platform. The demand for the product is stochastic isoelastic, which is given by

$D=y(p) \cdot \varepsilon$,

Where $y(p)$ is a deterministic and decreasing function of the $p$, and $\varepsilon$ is a random factor with general $\operatorname{CDF} F(\cdot)$ and $\operatorname{PDF} f(\cdot)$. Assume that $f(\cdot)$ has a support on $[A, B]$ with $0<A<B$, and $F(\cdot)$ satisfies the increasing generalized failure rate (IGFR) condition (see, e.g., Jiang and Wang 2010), i.e., $x f(x) /[1-F(x)]$ is increasing in $x$. We further assume that

$$
y(p)=a p^{-b},
$$

Where $a>0$ and $b>2$. $b$ Represents the price elasticity index of the (expected) demand (see, e.g., Jiang and Wang 2010).

The supplier considers to makes $R \& D$ investment to reduce its production cost. The value of $R \& D$ resources invested by the supplier is denoted by $k$. The production cost of the supplier is denoted by $c(k)$, which is a decreasing function of $k$, to reflect that $\mathrm{R} \& \mathrm{D}$ resources invested can result in a reduction in its production cost. We suppose that the supplier sells its product on the platform using consignment contract. Under such a contract, the supplier first chooses its production quantity $q$ and then sets its retail price $p$. For each unit sold, the platform keeps $r$ share of the sales revenue $p$ and the supplier obtain $1-r$ share of the sales revenue $p$.

The decisions to be made by the two players fall in the following three stages: In the first stage, the platform chooses the revenue share $r$; In the second stage, the supplier sets its production quantity $q$; In the last stage, the supplier sets its retail price $p$. All these decisions should be made before the selling season.

Based upon the above descriptions, the expected profits of the upstream supplier and the downstream platform can be written as follows, respectively:

$$
\pi_{u}(p, q)=(1-r) p E[\min (q, D)]-c(k) q-k,
$$

and

$$
\pi_{d}(r)=\operatorname{rp} E[\min (q, D)]
$$

In Eq. (3), $(1-r) p E[\min (q, D)]$ represents the expected revenue of the supplier by selling the product, $c(k) q$ corresponds to the production cost of the supplier, and $k$ is the R\&D investment cost of the supplier.

\section{Optimal Decisions of the Two Chain Members}

In this section, we investigate the optimal decisions of the entire supply chain. We derive the decisions in a backward manner. That is, we first consider the decision problem in the last stage, we 
then study the decision problem in second stage, we finally consider the decision problem in the first stage.

\subsection{Optimal Decisions in the Last Two Stages}

Following Jiang and Wang (2010), we define $z=q / y(p)$ as the stocking factor. Then the problem of decision making in the last and second stages is equivalent to choosing a retail price $p$ and a stocking factor $z$. Such a transformation can enable us to write the optimality condition more conveniently. Substituting $z=q / y(p)$ into Eq. (3), the expected profit of the supplier can be rewritten as

$$
\begin{aligned}
\pi_{u}(p, z) & =y(p)\{(1-r) p E[\min (z, \varepsilon)]-c(k) z\}-k \\
& =y(p)[(1-r) p h(z)-c(k) z]-k,
\end{aligned}
$$

Where $h(z)=E[\min (z, \varepsilon)]=z-\int_{A}^{z} F(x) d x$.

From Eq. (5), we can get the following result.

Proposition 1. For any revenue share decision chosen by the platform in the first stage, the optimal price in the last stage is given by

$$
p^{*}(r)=\frac{b c(k) z}{(b-1)(1-r) h(z)},
$$

And the optimal production decision in the second stage is given by

$$
q^{*}(r)=\frac{a(b-1)^{b}(1-r)^{b} h^{b}\left(z^{*}\right)}{b^{b} c^{b}(k)\left(z^{*}\right)^{b-1}}
$$

Where $z^{*}$ is determined by $(b-1) h\left(z^{*}\right)=b\left[1-F\left(z^{*}\right)\right] z^{*}$.

Proof. (i) Taking the first derivative of $\pi_{u}(p, z)$ with respect to $p$, we have

$$
\frac{d \pi_{u}(p, z)}{d p}=a p^{-b-1}[b z c(k)-(1-r)(b-1) p h(z)] \text {. }
$$

From Eq. (8), we can see that $\frac{d \pi_{u}(p, z)}{d p}$ is decreasing in $p$, i.e., $\frac{d^{2} \pi_{u}(p, z)}{d p^{2}}<0$. That is, $\pi_{u}(p, z)$ is concave in $p$, and thus the optimal retail price satisfies the first-order condition, i.e., $\frac{d \pi_{u}(p, z)}{d p}=0$, which yields Eq. (6).

(ii) Substituting $p^{*}(r)=\frac{b c(k) z}{(b-1)(1-r) h(z)}$ of Eq. (6) into Eq. (5), we can show that

$$
\pi_{u}(z)=\frac{a(b-1)^{b-1}(1-r)^{b} h^{b}(z)}{b^{b} c^{b-1}(k) z^{b-1}}-k .
$$

From Jiang and Wang (2010), we know that $\frac{h^{b}(z)}{z^{b-1}}$ achieves its maximum at $z^{*}$, which is uniquely determined by $(b-1) h\left(z^{*}\right)=b\left[1-F\left(z^{*}\right)\right] z^{*}$. We thus complete the proof of Proposition 1 .

Proposition 1 characterizes the optimal response decisions in the last two stages. From Proposition 1 , we can see that if a higher revenue share is chosen by the platform, then a higher retail price and a lower production quantity will set by the supplier. Next, we consider the decision problem in the first stage.

\subsection{Optimal Decisions in the First Stage}

Knowing that the supplier chooses its retail price and production quantity according to Eq. (6) and Eq. (7), the platform chooses a revenue to maximize its expected profit. 
Now, substituting $p^{*}(r)=\frac{b c(k) z}{(b-1)(1-r) h(z)}$ of Eq. (6), $q^{*}(r)=\frac{a(b-1)^{b}(1-r)^{b} h^{b}\left(z^{*}\right)}{b^{b} c^{b}(k)\left(z^{*}\right)^{b-1}}$ of Eq. (7) and $z=q / y(p)$ into Eq. (4), we can rewritten the expected profit of the platform as

$$
\pi_{d}(r)=\frac{a(b-1)^{b-1} h^{b}\left(z^{*}\right)}{b^{b-1} c^{b-1}(k)\left(z^{*}\right)^{b-1}} r(1-r)^{b-1} \text {. }
$$

This allows us to have the following result.

Proposition 2. Knowing the supplier's optimal response pricing and production decisions, the optimal revenue share of the platform in the first stage is given by

$$
r=\frac{1}{b}
$$

Proof. Taking the first derivative of $\pi_{d}(r)$ with respect to $r$, we have

$$
\frac{d \pi_{d}(r)}{d r}=\frac{a(b-1)^{b-1} h^{b}\left(z^{*}\right)}{b^{b-1} c^{b-1}(k)\left(z^{*}\right)^{b-1}}(1-r)^{b-2}(1-r b) \text {. }
$$

Because $0<r<1$, we know from Eq. (12) that: If $0<r<\frac{1}{b}$, then $\frac{d \pi_{d}(r)}{d r}>0$; If $\frac{1}{b}<r<1$, then $\frac{d \pi_{d}(r)}{d r}<0$. That is, $\pi_{d}(r)$ is unimodal in $r$, and thus the optimal revenue share satisfies the first-order condition, i.e., $\frac{d \pi_{d}(r)}{d r}=0$, which yields Eq. (11).

Proposition 2 shows the optimal revenue share for the platform is independent of the supplier's production cost. This may partly explain the phenomenon that the platform usually sets the same revenue share for different substitutable suppliers. In addition, substituting and $z=z^{*}$ and $r=\frac{1}{b}$ of Eq. (11) into Eq. (6) and Eq. (7), respectively, we can show that the optimal retail price and the production quantity are as follows:

$$
p^{*}=\frac{b^{2} c(k) z^{*}}{(b-1)^{2} h\left(z^{*}\right)}
$$

And

$$
q^{*}=\frac{a(b-1)^{2 b} h^{b}\left(z^{*}\right)}{b^{2 b} c^{b}(k)\left(z^{*}\right)^{b-1}} .
$$

Moreover, substituting and $z=z^{*}$ and $r=\frac{1}{b}$ of Eq. (11) into Eq. (5) and Eq. (10), respectively, we can show that for any R\&D investment strategy chosen by the supplier, the corresponding expected profits for the supplier and the platform are as follows:

$$
\pi_{s}=\frac{a(b-1)^{2 b-1} h^{b}\left(z^{*}\right)}{b^{2 b} c^{b-1}(k)\left(z^{*}\right)^{b-1}}-k,
$$

And

$$
\pi_{d}=\frac{a(b-1)^{2 b-2} h^{b}\left(z^{*}\right)}{b^{2 b-1} c^{b-1}(k)\left(z^{*}\right)^{b-1}} .
$$

From Eq. (13), Eq. (14), Eq. (15) and Eq. (16), we can get the following result.

Proposition 3. (i) When the supplier makes R\&D investment to reduce its production cost, the retail price decreases and the production quantity increases. (ii) The platform can always benefit from the supplier's R\&D investment, while the supplier may not benefit from the investment.

Proof. Proposition 3 follows directly from the fact that $c(k)$ is a decreasing function of $k$. We thus complete the proof of Proposition 3. 
Proposition 3(i) indicates that the supplier's R\&D investment can lead to a lower retail price and a higher production quantity. This is not surprising, since the supply chain becomes more cost efficient. Additionally, Proposition 3(ii) implies that the platform hopes that the supplier conducts R\&D investment, while the supplier may not conduct R\&D investment. This is because the supplier should weigh up the benefit caused by the R\&D investment and the corresponding investment cost, and then determines whether to invest. Specifically: if and only if

$$
\left(\pi_{s}\right)-\left(\left.\pi_{s}\right|_{k=0}\right)=\frac{a(b-1)^{2 b-1} h^{b}\left(z^{*}\right)}{b^{2 b}\left(z^{*}\right)^{b-1}}\left[\frac{1}{c^{b-1}(k)}-\frac{1}{c^{b-1}(0)}\right]-k>0,
$$

Then the supplier should invest, where $\frac{a(b-1)^{2 b-1} h^{b}\left(z^{*}\right)}{b^{2 b}\left(z^{*}\right)^{b-1}}\left[\frac{1}{c^{b-1}(k)}-\frac{1}{c^{b-1}(0)}\right]$ represents the benefit from R\&D investment for the supplier.

\section{Concluding Remarks}

This paper is motivated by the increasing popularity of platform sale, where the suppliers usually sell their products on the platform using consignment contract; and also motivated by the fact that many products have constant price elasticities (for example, price elasticity for Chevrolet automobiles is 4.0, see, e.g., Anderson and McLellan 1997).

This paper considers the problem of the supplier's R\&D investment in the context of the above environment. By establishing a three stage Stackelberg game, the paper derives the optimal pricing and production decisions for the supplier and the platform. Then, the paper analyzes the impact of the supplier's R\&D investment on supply chain operations.

In this paper, we assume that each chain member is risk neutral. In future research, it is interesting to extend our risk neutral model into risk-averse model.

\section{Acknowledgements}

This paper is supported by the National Natural Science Foundation of China (No. 70772068).

\section{References}

[1]. Grossmann I. E. (2004). Challenges in the new millennium: product discovery and design, enterprise and supply chain optimization, global life cycle assessment. Computers \& Chemical Engineering, 29(1), 29-39.

[2]. Zhang H., Hong D. (2017). Manufacturer's R\&D Investment Strategy and Pricing Decisions in a Decentralized Supply Chain. Discrete Dynamics in Nature and Society, 2017, 1-10.

[3]. Ge Z., Hu Q., Xia Y. (2014). Firms’ R\&D cooperation behavior in a supply Chain. Production and Operations Management, 23(4), 599-609.

[4]. Usta M., Erhun F., Hausman W. H. (2014). Supply licensing when the manufacturer strategically commits to invest in R\&D. Naval Research Logistics, 61(4), 341-350.

[5]. Banerjee S., Lin P. (2001). Vertical research joint ventures. International Journal of Industrial Organization, 19(1), 285-302.

[6]. Banerjee S., Lin P. (2003). Downstream R\&D, raising rivals’ costs, and input price contracts. International Journal of Industrial Organization, 21(1), 79-96.

[7]. Bernstein F., Kök A. G. (2009). Dynamic cost reduction through process improvement in assembly networks. Management Science, 55(4), 552-567.

[8]. Bernstein F., Kök A. G., Meca A. (2015). Cooperation in assembly systems: The role of knowledge sharing networks. European Journal of Operational Research, 240(1), 160-171.

[9]. Ishii A. (2004). Cooperative R\&D between vertically related firms with spillovers. International Journal of Industrial Organization, 22(8), 1213-1235. 
[10]. Gupta S. (2008). Research note-channel structure with knowledge spillovers. Marketing Science, 27(2), 247-261.

[11]. Leng M., Li Z., Liang L. (2016). Implications for the role of retailers in quality assurance. Production and Operations Management, 25(5), 779-790.

[12]. Seifbarghy M., Nouhi K., Mahmoudi A. (2015). Contract design in a supply chain considering price and quality dependent demand with customer segmentation. International Journal of Production Economics, 167, 108-118.

[13]. Chen J., Liang L., Yang F. (2015). Cooperative quality investment in outsourcing. International Journal of Production Economics, 162, 174-191.

[14]. Zhu W., He Y. (2017). Green product design in supply chains under competition. European Journal of Operational Research, 258(1), 165-180.

[15]. Yu J., Ma S. (2013). Impact of decision sequence of pricing and quality investment in decentralized assembly system. Journal of Manufacturing Systems, 32(4), 664-679.

[16]. Anderson P. L., McLellan R. D., Overton J. P., Wolfram G. L. Price elasticity of de- mand. Mackinac center for public policy. 1997. http://www.mackinac.org/1247 .

[17]. Jiang L., Wang Y. (2010). Supplier competition in decentralized assembly systems with price-sensitive and uncertain demand. Manufacturing \& Service Operations Management, 12(1), 93-101. 